\title{
The Digital Radiography of Archaeological Pottery: Program and Protocols for the Analysis of Production
}

\author{
A. F. Greene ${ }^{\mathrm{a}, *}$, C. W. Hartley ${ }^{\mathrm{b}}$, P. N. Doumani Dupuyc,d, M. Chinander ${ }^{\mathrm{e}}$ \\ ${ }^{a}$ Stanford University, Department of Anthropology, Stanford Archaeology Center, PO Box 20446, Stanford, CA 94309 \\ ${ }^{b}$ University of Chicago, Department of Anthropology, 1126 E 59th Street, Chicago, IL 60637 \\ ${ }^{c}$ Washington University in St. Louis, Department of Anthropology, Campus Box 1114, McMillan Hall, Room 112, 1 \\ Brookings Drive, St. Louis, MO 63130 \\ ${ }^{d}$ Christian-Albrechts-Universitat zu Kiel, Institute of Pre- and Protohistoric Archaeology, Johanna-Mestorf-Strasse 2-6, \\ 24118, Kiel, Germany (Present Address) \\ ${ }^{e}$ University of Chicago, Department of Orthopaedic Surgery and Rehabilitation Medicine, Duchossois Center for Advanced \\ Medicine, 5841 South Maryland Avenue, MC 3079, Chicago, 60637
}

\begin{abstract}
Archaeologists collaborating with material scientists at Argonne National Laboratory (ANL) as part of the Making of Ancient Eurasia (MAE) Project have developed formal methodological standards for the assemblage-based digital radiographic (DR) analysis of archaeological pottery. While analog radiography of pottery (X-radiography, Xeroradiography, etc.) has functioned as a common disciplinary tool for some time, inaccessibility, obsolescence, and significantly enhanced functionality have made DR instrumentation increasingly attractive and vital. This article presents the theoretical underpinnings, technique development, and resultant protocols that allow digital radiography to analyze very large assemblages and provide quantitative data sets that act as true counterparts to geochemical and mineralogical ones. As a technique of structural pottery evaluation, DR is particularly suited to the analysis of ceramic paste preparation and vessel formation, providing lines of evidence that can flesh out neglected portions of the chaîne opératoire, augment existing geochemical or typological classifications, and help more deeply characterize various potting traditions. Such datasets are most useful to scholars interested in harnessing the ability of the pottery "life cycle" to shed light on economic life, learning frameworks, and human social differences and group identities. The technical capacities and analytical potential of DR are demonstrated through several test analyses of ancient Chinese pottery, to be followed by more extensive case studies in draft. Prospects for closely related, three-dimensional X-ray computed tomographic approaches are also discussed.
\end{abstract}

Keywords: digital radiography, image analysis, archaeological pottery, technology, assemblage-based archaeological science, structural materials analysis, Eurasia

\footnotetext{
This document is a collaborative effort.

*Corresponding author

Email addresses: afgreene@stanford.edu (A. F. Greene), chartley@uchicago.edu (C. W. Hartley), pauladoumani@wustl.edu (P. N. Doumani Dupuy), mchinand@uchicago.edu (M. Chinander)
}

July 13, 2016

(C) 2016. This manuscript version is made available under the Elsevier user license 
1987, 1988). As a result, X-ray approaches to imaging ceramic materials are among the most developed and deeply detailed (Berg, 2008; Braun, 1982; Carr, 1993; Carr and Riddick, 1990; Ellingson et al., 1988; Middleton, 2005), having been particularly productive in the structural examination of paste preparation and vessel formation, analytical foci that have received diminished attention as the discipline has honed its compositional approaches (cf. Jakes, 2002; Lambert, 1997; Pollard and Heron, 2008). Digital radiographic and computational tools are now well positioned to vastly enhance the capacity to perform structural pottery analysis by enabling high volume $(\mathrm{n} \geq 300)$, more quantitative research to take precedence over the use of smaller, less "representative" (cf. Shennan, 1997: 364), and qualitative datasets (Ellingson et al., 1997; Greene and Hartley, 2009; Kahl and Ramminger, 2012; Vandiver et al., 1991). This article discusses systematic digital radiographic protocols developed for such purposes at Argonne National Laboratory (ANL) and demonstrates both the variety of expanded analyses made possible by the method and their broader archaeological implications (see Greene, 2013; Greene, et al. n.d., and Doumani, 2014 for case studies applying these methods that are more extensive than the example analyses outlined below).

In the 1970s and 80s, radiographic approaches made significant contributions to pottery analysis by permitting advancement of multiple sophisticated frameworks for characterizing fabrics (Braun, 1982; Carr, 1993; Carr and Riddick, 1990), and by allowing examination and identification of formation techniques through comparative analysis of void orientations and paste gradients as viewed in film and Xeroradiographic paper imagery (Carr and Riddick, 1990; Foster, 1985; Glanzman and Fleming, 1986; Rye, 1977; Vandiver, 1987, 1988; Vandiver et al., 1991). ${ }^{1}$ By developing, through iterative experimentation and analysis, diagnostic models for analyzing the size, shape, and orientation of fabric components such as inclusions and pores and for recording joins between vessel sub-parts (coils, slabs, applied features, etc.), these scholars made possible new perspectives on vessel structure that provided critical evidence for the analysis of production techniques and sequences. Such radiographic technique development and materials analysis has waned, however, as obsolescence and lack of support has left analog tools difficult to access. In addition, slower data acquisition speeds and duplicative digitization workflows ignore the two primary benefits of DR: its ability to provide high volume, assemblage-based $(\mathrm{n} \geq 300)$ data and to enable that data to be qualitatively and quantitatively coded directly into archaeological databases and cross-analyzed across typological, spatial/contextual, and landscape evidence. Historically, pottery radiography by scholars such as Braun, Carr, Heinsch, and Vandiver, focused on samples of a maximum of several hundred potsherds, with Carr's Woodland Americas study standing as the most extensive by far at over 3,000. In contrast, our DR research has collected more than 4,000 radiographs of almost 2,000 distinct potsherds and vessels in a relatively restricted number of data collection events, permitting as many as 200 images to be taken in a single 8-hour work day. Protocols and software for digital data collection, post-processing, and image analysis can therefore make substantial contributions to this important method, inject renewed vigor into its practice, and reorient the instrumental analysis of archaeological pottery to more comprehensively and nondestructively analyze production.

Large format X-ray systems often referred to as "experimental" or "industrial" radiography equipment (Quinn and Sigl, 1980) draw our primary focus here. They are most useful for the systematic digital radiography of archaeological materials for multiple reasons. First, ubiquitous medical radiographic equipment relies on low energy levels designed for use on human tissues, which insufficiently penetrate ceramic materials. In addition, restrictions on the size and shape of pottery that can be inserted into the closed cabinet systems and sample holders of medical instrumentation negate the methods non-destructivity by requiring potsherds or vessels to be cut or broken. In contrast, industrial systems lack significant sample size restrictions, can employ a broader range of X-ray energies (peak kilovoltage) and greater levels of current (milliamperes), and share a more "open" setup for component manipulation. Such systems therefore enable the examination of large assemblages and rare, unusually shaped, or "oversized" objects.

Of course, data collection protocols and analytical standards associated with industrial X-ray systems, like those of their medical counterparts, have a traditionally narrow focus: they have been designed to examine materially uniform, modern substances and objects in which homogeneity and lack of structural anomalies are valued. ${ }^{2}$ Significant experimentation and testing has therefore been necessary to apply indus-

\footnotetext{
${ }^{1}$ Research in the radiography of archaeological pottery has also resulted in the development of a number of secondary methods, studying issues such as: distinguishment between wheel-thrown and wheel-coiled vessels (Berg, 2011), mineral identification (Berg, 2008; Carr and Komorowski, 1995; Foster, 1985), and affiliation of potsherds with parent vessels (Carr, 1993).

${ }^{2}$ Much industrial radiography is focused on non-destructive evaluation (NDE) and quality control of mass-produced com-
} 
trial instrumentation to more heterogeneous and less materially standardized archaeological objects. Within these new parameters, the method is able to both replicate the effective results of various analog approaches (X-radiography, Xeroradiography, etc., see Greene and Hartley, 2009: Figure 5d and Section 3.3 below) by applying statistical manipulation (recursive histogram stretching, normalization, etc.), and push analysis beyond the range of earlier techniques through the application of algorithmic filters that identify features and matrix patterns imperceptible to the human eye. (While humans can distinguish several dozen shades of gray [Kreit et al., 2013], digital image capture devices can collect data with over 65,000 .) The sections that follow describe instruments, data acquisition protocols, software tools, and interpretive frameworks developed to apply digital radiography to the mass analysis of archaeological pottery and to pose questions regarding long-standing typological or geochemical models, neglected portions of the chaîne opératoire, and detailed understandings of individual pottery traditions writ large. Examples from the analysis of formation and paste preparation in Longshan pottery from the site of Huizui, China help to illustrate the new utility of this instrumentation. A separate paper in preparation (Greene et al., n.d.) demonstrates the application of these protocols in three distinct, assemblage-based Eurasian case studies.

\section{X-radiation and Radiography: Properties, Practices, and Affordances}

\subsection{Basic Principles of X-radiographic Physics}

As is true for photography, where digital cameras are now ubiquitous, digital radiography has largely replaced conventional film radiography in medicine (Brennan, 2002; Bushberg et al., 2002; Herring, 2011), industry (Boyce and Samei, 2006; Ellingson et al., 1997), conservation (Lang and Middleton, 2005), and, increasingly, in archaeology (Casali, 2006; Lang, 2005; Middleton, 2005; Vandiver et al., 1991). Most aspects of collecting digital and analog radiography are identical, but the digital nature of the resultant data sets and their amenability to post-processing and manipulation make digital instrumentation more productively suited to the mass, assemblage-based analysis of archaeological materials. Of particular utility is the ability of digital imagery to be filtered and manipulated as quantitative data sets, producing second and third-order statistical analyses that convert 16-bit raw data into contrast-enhanced feature maps. This section reviews principles common to both analog and digital radiography, followed by an outline of the primary, X-ray detection-based distinctions between these two types of X-radiographic instrumentation.

As its name implies, radiography is the process of using electromagnetic radiation (radio-) to create images (-graphy). In contrast to photography, which measures reflected light, the property measured in radiography is the amount of X-ray beam attenuated by the object as it passes through. Attenuation refers to loss of X-ray photons, through absorption or scattering, as they move through objects (Bushberg et al., 2002: 45). This depends on composition and thickness of the object, as well as energy, or more appropriately, the energy spectrum (wavelength, frequency) of the X-ray beam. Relationships between these parameters are described by the Beer-Lambert's law exponential equation outlined in Equation 1 (Casali, 2006: 52-53):

$$
I=I_{o} e^{-\frac{\mu e}{\rho} \rho t}
$$

where $I_{o}$ is the initial intensity (number of X-ray photons) of the X-ray beam entering the object, $I$ is the intensity of the beam exiting the object, $\frac{\mu \mathrm{e}}{\rho}\left(\mathrm{mm}^{2} / \mathrm{g}\right)$ is the mass attenuation coefficient of the material for X-rays of energy $e, \rho$ is the density of the object $\left(\mathrm{g} / \mathrm{mm}^{3}\right)$, and $t(\mathrm{~mm})$ is its thickness. In general, elements with higher atomic numbers have higher mass attenuation coefficients at a given X-ray energy and attenuation decreases with increasing X-ray energy.

For objects composed of several different materials or densities and for a given spectrum of X-ray energies, Equation 1 can be expanded to a linear combination of relevant variables. Figure 1a depicts a typical radiographic setup (in place at ANL's X-ray Computed Tomography laboratory) with an example X-ray beam emanating from the X-ray tube ("source"), passing through the object, and then incident onto the recording medium. According to Beer's law (see above), the intensity of the X-rays incident onto the recording medium is proportional to their number and energy, depends on how much they have been attenuated by the

modities and machine components (Ellingson et al., 1997; Liaptsis et al., 2010; Mei et al., 2007; Sun et al., 2008). See Hellier 2012, as well as the journals Research in Nondestructive Evaluation, Nondestructive Testing and Evaluation, and the Journal of Nondestructive Evaluation for an introduction to this materials analytical sub-field. 
material through which they have passed, and and is measured in terms of "exposure" (units: Roentgens) (Bushberg et al., 2002; Casali, 2006). For direct X-ray radiography, X-rays expose the recording medium and the resulting optical density (or grayscale value) depends on X-ray energy and intensity of the exposure. An intensifying screen is typically employed in medical radiography in order to (pre-)absorb the X-rays and produce visible light photons that expose the film (Carr and Riddick, 1990: 47-49; Curry III et al., 1990). ${ }^{3}$ An intensifying screen requires much less $\mathrm{X}$-ray exposure but conversely, it has lower spatial resolution (see this same section, below).

An analyst must adjust several imaging parameters to control radiograph quality. These include Xray energy, also called "peak kilovoltage" $(\mathrm{kVp})$, X-ray tube current (milliamperes, $\mathrm{mA}$ ) and exposure or "integration" time (seconds, IT) (often combined into a single parameter called mAs which is the product of the two), and imaging geometry: the X-ray source-to-detector distance (SDD) and object-to-detector distance (ODD). The quality of images collected using specific data acquisition parameters is assessed using three factors: noise, contrast, and resolution. In medical radiography, due to concerns for radiation dose to patients (and the need for short exposure times to minimize image blurring if the patient moves), the predominant source of noise is the relatively few X-ray photons used during image acquisition, where noise is proportional to the number of X-rays (Poisson distributed [Bushberg et al., 2002: 156-157]). Since neither radiation dose nor object motion are concerns for archaeological radiography, noise can be minimized with exposures relying on higher numbers of X-ray photons, leaving image contrast and (spatial) resolution as primary image quality factors to be considered and potentially optimized.

Image contrast is a measure of the difference of optical densities or gray-levels of neighboring regions of an image (Bushberg et al., 2002; Casali, 2006). High image contrast greatly assists identification and delineation of structural features in a specific region of interest. Contrast is first determined by the intrinsic material properties of the sample (chemical makeup, density, shape and structure), which are responsible for its differential X-ray attenuation. The selection of the X-ray peak energy $(\mathrm{kVp})$ affects object contrast since materials have different X-ray attenuation depending on X-ray photon energy. Resulting contrast therefore depends on the object's material properties and on the recording medium's response to the incident X-ray exposure. For conventional analog radiography, the relationship between the exposure to film and resulting optical density on film is linear over only a limited range of exposure values and has a sigmoidal shape (Bushberg et al., 2002: 158). In low and high exposure regions, little difference occurs in the optical density of film for a given difference in exposure. Thus, the analyst must carefully select a beam current and exposure (mAs) setting where exposure to the film falls within the linear region. On the other hand, digital detectors have a linear response to exposure across a wider range, so choice of exposure setting is less critical (Bushberg et al., 2002: 260).

Spatial resolution, also essential to image quality, measures how small of an object can be distinguished in the radiographic image (Bushberg et al., 2002; Casali, 2006). An imaging system with higher spatial resolution can distinguish between smaller features or sub-structures than a system with lower spatial resolution. Factors that affect spatial resolution include X-ray tube focal spot size, type and thickness of the intensifying screen, and the qualities of the detection device. Of these three components, the intensifying screen has the lowest spatial resolution, and thus will have greatest impact on the system's overall spatial resolution.

Spatial resolution of a given system can be determined by measuring either the maximum number of line pairs discernible when imaging a specially designed "phantom" or the modulation transfer function (MTF) through an assessment of the edge sharpness in an image of a thin object (Samei et al., 1998). MTF is a measurement of how well an imaging system records all spatial frequencies - measures of recurrence of the sinusoidal components in a given structure - in the image. An ideal imaging system would have an MTF value of "one" for all spatial frequencies. For real-world imaging systems, however, MTF values decrease with increasing spatial frequency (i.e., smaller structures and details).

"Effective" resolution of the imaging system (i.e., the dynamic millimeters represented by a single detector pixel) can be increased by changing the distance between object and film (or detector), so the image becomes magnified. Magnification factor, $\mathrm{M}=\mathrm{I} / \mathrm{O}$, is given by $\mathrm{SDD} / \mathrm{SOD}$ where $\mathrm{SDD}$ is the source-to-detector distance and SOD is the source-to-object distance (see Figure 1a). However, increased magnification does

\footnotetext{
${ }^{3}$ Choice of intensifying screens depends on the specific X-ray energy employed and the desired balance between noise and resolution. The protocols and testing described in Section 3 used no screens so as to maximize resolution.
} 


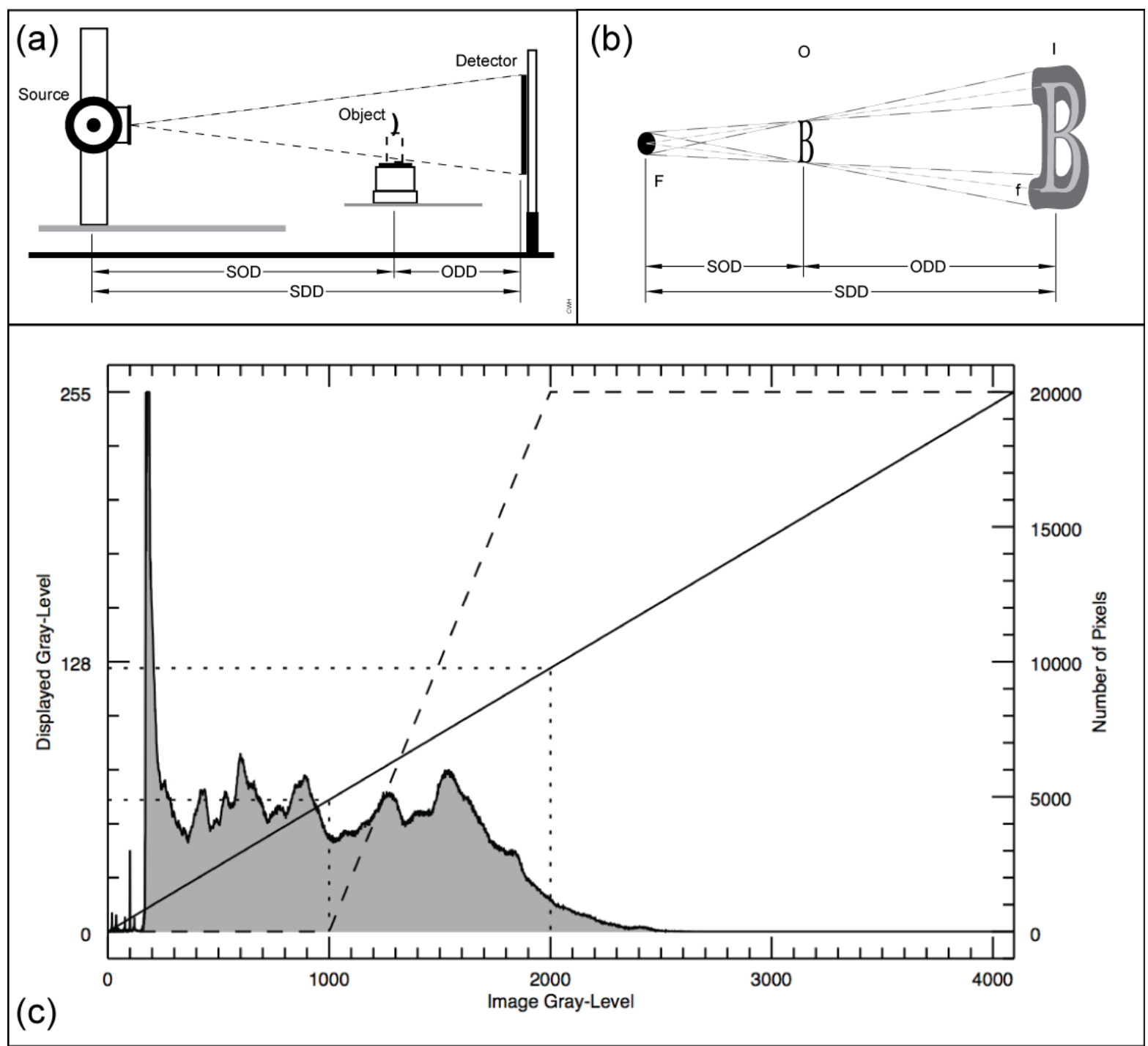

Figure 1: (A) Radiographic setup at the Argonne National Laboratory X-ray Computed Tomography facility showing the relationship between the X-ray source, sample object, and digital detector. The source and sample stage can translate horizontally, while the sample stage can also translate in the $\vartheta$ (rotational) direction; (B) The magnification and "edge spread" effects of a typical radiographic setup, where magnification $\mathrm{M}=\mathrm{I} / \mathrm{O}$, is given by SDD/SOD where SDD is the source-to-detector distance and SOD is the source-to-object distance. The object's edge, here the boundary of a theoretical letter "B," is spread to a width of $f$, compared to being infinitesimally small if the focal spot size (F) was a single point; (C) Graph of the dynamics of the "windowing" process, by which the gray-levels of the image are linearly mapped to the gray-level range of $0-255$. Windowing can increase the displayed contrast of regions of interest by including only those gray-levels distinguishable to the human eye. 
not improve spatial resolution in all cases because blur generated by the size of the X-ray tube focal spot increases in concert. This is often termed "geometric unsharpness" since by the geometry of similar triangles, it can be shown that moving the object closer to the focal spot increases blur due to the focal spot's finite size (i.e., it is not a point source). This fact is demonstrated in Figure 1b, where the object's edge is spread to a width of $\mathrm{f}=\mathrm{F}(\mathrm{M}-1)$, where $\mathrm{f}$ is the spread edge of the imaged object, $\mathrm{F}$ is the size of the focal spot of the X-ray tube, and $\mathrm{M}$ is magnification factor (see above). (If the focal spot constituted a single point, the object's edge would be spread an infinitesimally small amount.) This can lead to a lower MTF for some spatial frequencies at higher magnification and is important for identifying optimal imaging settings (Boyce and Samei, 2006).

\subsection{Digital and Analog Data Collection Technologies}

Digital radiography systems are virtually identical to conventional analog systems, except for the technology of X-ray detection employed. Instead of X-rays being incident onto film (or intensifying screen), they are incident onto a digital detector, producing an image that can be immediately stored on a computer. Several digital radiographic detector systems exist, including computed radiographic (CR) and direct and indirect digital radiographic systems (Bushberg et al., 2002; Casali, 2006). These different detection methods create a digital image from the attenuated X-rays, consisting of a rectangular grid of pixels with a gray-level proportional to the exposure of their specific portion of the detector. In a digital image, gray-level is recorded at discrete steps (e.g., integers 0-255) instead of the continuous range (infinitely small steps) of optical density for film (Lang and Middleton, 2005). The number of gray-levels is determined by the bit-depth of the image, typically 8- (256 shades of gray), 12- (4,096 shades of gray), or 16-bits $(65,536$ shades of gray) in digital radiography. Bit depth is the number of bits (in binary 0 or 1 values) used to store a single pixel's gray-level. The number of gray levels for a bit-depth $\mathrm{N}$ is $2^{\mathrm{N}}$, so an 8-bit image has $2^{8}=256$ gray-levels. Increasing bit-depth allows one to distinguish between smaller exposure differences (finer contrast distinctions) in the image (Casali, 2006).

In conventional analog radiography, the detection device (i.e., film, Xeroradiographic paper, etc.) is also the storage and display device, although the analyst is unable to modify or quantify revealed features or regions of interest without using digital tools. In digital radiography, detection, storage, and display utilize separate devices (i.e., detector, hard-drive, and monitor), which allows each step to be optimized independently. Unlike film and paper, digital detectors also have a linear response to exposure over a much larger range of values, while the digital nature of the data makes storage, preservation, and replication of image files for publication much more efficient and suited to long term, iterative research. Most importantly, computational tools allow for the manipulation of digital radiographs, permitting the analyst to measure features systematically, create second and third order imagery, and statistically analyze the large, multivariate data sets that result (Casali, 2006; Greene and Hartley, 2009). Some of these manipulations include magnification, image enhancement (histogram equalization), and windowing, which determines how image gray-levels are mapped to display gray-levels. This is particularly useful for high bit-depth images, since most softwares map image gray-levels to the range of 0-255 (solid line in Figure 1c) to accommodate standard monitors. (Gonzalez and Woods, 2007; Ishida et al., 1984). If regions of interest in a given image fall within a limited range of image gray-levels, they will only use a small range of the displayed gray-level range (see the dotted lines in Figure 1c) and have a low level of contrast when displayed. By changing the windowing to include only relevant gray-levels (see the dashed line in Figure 1c, outlined further in Section 3), one can increase the displayed contrast of regions of interest in the image. These functions, among others, are available in most image display software (Gonzalez and Woods, 2007) as well as our custom analysis program, Sherd Image Viewer and Analysis (ShIVA), described in Sections 3 and 4 below.

\subsection{A Short Note on X-ray Computed Tomography}

While radiography allows non-invasive observation of internal features that would otherwise be hidden, the projectional nature of the method (i.e., the creation of two-dimensional images from three-dimensional objects) leaves it prone to obfuscating microstructures and their relationships in the depth dimension (perpendicular to the imaging plane). Thus, structures that overlie each other in this dimension can obscure one another and lead to either incorrect interpretation (e.g., two distinct overlapping structures appearing to be a single structure) or complete undetectability (Bushberg et al., 2002; Casali, 2006; Lang and Middleton, 2005). X-ray computed tomography is a radiographic imaging modality that can overcome these deficiencies 
by creating three-dimensional images of an object using a similar digital radiographic system. This is accomplished by taking multiple images from a series of systematically configured, circumferential angles and then reconstructing "slices" of the object via canonical CT algorithms (cone-beam, fan-beam, etc.), often referred to as "back projection" (see Casali, 2006 and Bushberg et al., 2002 for in-depth discussions of XCT theory). The resultant slices can be stacked sequentially to create a three-dimensional image, or "volume." In practice, images are typically acquired by placing the object on a rotational stage between a fixed X-ray source and detector and repeating a "step-and-shoot" process where the stage is rotated a small amount between each image acquisition (Bushberg et al., 2002: 329). ${ }^{4}$ While XCT protocols and image quality testing for archaeological pottery remain in development (see Applbaum and Applbaum, 2005; Casali, 2006; Kahl and Ramminger, 2012; Lang and Middleton, 2005; Vandiver et al., 1991), systematic imaging protocols, post-processing routines, and analytical software for the digital radiography of archaeological potsherds and vessels prepared by MAE collaborators are ready for common use and dissemination.

\section{Digital Radiography Technique Development for Archaeological Pottery}

The MAE Project has operated the majority of its radiographic technique development and materials analysis out of Argonne National Laboratory (ANL) since 2005, when research was initiated under a collaborative seed grant between the University of Chicago and the ANL Board of Governors. The ANL non-destructive evaluation (NDE) ceramic sub-group focuses on evaluation of industrial aluminum-silicate materials (Ellingson et al., 1988; Vandiver et al., 1991), providing a productive source of collegial expertise. In addition, NDE assembled a state-of-the-art experimental radiography lab for DR and XCT of industrial ceramics in the early 2000s, a finely tunable set of instruments that serves as our analytical base up to the present.

\subsection{Laboratory Facilities and Operating Procedures}

The NDE X-ray computed tomography (XCT) laboratory features several multimodal instruments, by which is meant they posses the ability to analyze objects from: (1) multiple scalar perspectives and (2) multiple instrumental platforms (Greene In Press; Greene and Hartley, n.d.). The facilities are split across two instrument bays, controlled from a central workspace. Unlike commercial "closed" DR systems, this is a fully modular industrial radiography setup, much less restricted by sample size or geometry than cabinetbased systems. The primary experimental radiography system features both IRT and Philips X-ray tubes, but the Philips is primarily utilized, with a maximum $\mathrm{kVp}$ of 420 . Several digital detectors are available, but two are most relevant for high volume DR of archaeological pottery: Perkin Elmer area detectors with pixel sizes of 400 microns and 200 microns, respectively. These detectors utilize gadolinium oxysulfide (GOS) photoconductors to convert X-ray photons to optical photons and thin-film transistor (TFT) arrays to read out the signal. A second bay features an IRT HOMX 161 micro-focus tube that is compatible with both aforementioned detectors. The micro-focus instrument is most useful for detailed investigation of specific particles, voids, or microstructures on the level of tens of microns (see Kahl and Ramminger, 2012 for a detailed example of the microradiographic and microtomographic analysis of archaeological pottery).

\subsection{Development of Data Acquisition Protocols}

The experimental, modular setup in the XCT laboratory allows digital radiography to be acquired through manipulation of various instrument and sample (object) positions (see Figure 1). In the primary bay, the setup includes a sample stage that translates in the $\mathrm{x}$, y and $\vartheta$ (rotational) directions, an X-ray tube ("source") that translates in $\mathrm{x}$ and $\mathrm{y}$ directions, and a fixed rack for securing various detectors. Each translating stage can be finely controlled through Fortran-based software, allowing adjustment at a micrometer scale of the spatial relationships between sample, source, and detector. By coordinating the setup through nine standard positions (see Table 1), scale and magnification of the acquired radiographic imagery can be closely adjusted so that the system collects imagery of the most analytically useful quality. For example, while in position 7 $(\mathrm{SDD}=219 \mathrm{~cm}, \mathrm{SOD}=95 \mathrm{~cm})$, the magnification $(231 \%)$ and effective pixel size for the ANL detectors $(0.0868$

\footnotetext{
${ }^{4}$ In more elaborate medical applications, the X-ray source and detector are rotated around the sample or body being imaged (see Bushberg et al., 2002: 337).
} 
$\mu \mathrm{m}$ for the $200 \mu \mathrm{m}$ detector, $0.1735 \mu \mathrm{m}$ for the $400 \mu \mathrm{m}$ detector) will frame objects that fit an area up to 17 centimeters square. Larger objects are therefore imaged in positions 5 or 6 (see Table 1), where source and sample are farther apart and the effective size of the detector is between 27 and 33 centimeters square.

As noted in the prior section, X-ray beam peak kilovoltage $(\mathrm{kVp})$, milliamperes $(\mathrm{mA})$, and integration time (IT) also undergo adjustment in order to acquire imagery of desired contrast and optimum quality. IT is controlled through proprietary data acquisition software, while direct control knobs allow the operator to adjust $\mathrm{kVp}$ and $\mathrm{mA}$. Between 2005 and 2010 the MAE project developed protocols for these various geometries and energy settings in order to optimize the image quality of pottery radiographs (Greene and Hartley, 2009)..$^{5}$

Analog film radiography (X-radiography) and Xeroradiography of pottery utilized long integration periods, requiring artifacts to be exposed to a relatively low $\mathrm{kVp}$ X-ray beam to allow the film or paper to absorb photons for many minutes (Carr and Riddick, 1990; Lang and Middleton, 2005). Digital components, however, achieve high contrast with comparatively short exposure periods, which also extend the system's lifespan (Greene and Hartley 2009; see also the portion of Section 2.1 on "exposure" above). Moreover, digital detectors with long analog-style integration times are uncommon, and result in underexposure at relatively low $\mathrm{kVp}$, seen in Figure 2c, as well as too much attenuation of the source: a perfect recipe for overly dark imagery. Thus, it was essential to determine specific ranges $\mathrm{kVp}, \mathrm{mA}$, and IT more amenable to systematic digital X-ray system research, and to develop a technique for measuring image quality across a large-scale dataset. ${ }^{6}$

Table 2 summarizes several of the more relevant settings combinations tested for ideal geometry, energy, and exposure length parameters. Voltage/ampere combinations are essentially infinite in number; therefore settings were selected at $100 \mathrm{kVp}$ intervals (i.e., 100, 200,300, and $400 \mathrm{kVp}$ ) and paired with different sets of $\mathrm{SDD}$, SOD, and integration time. Our testing identified the $\mathrm{mA}$ settings that produced viable images (Figure $2 \mathrm{~b}$ shows one such appropriately acquired image) when paired with these $\mathrm{kVp}$ settings (i.e., images that showed neither energy "saturation" of the detector, nor over-attenuation of the X-ray beam, as demonstrated in the poor quality radiographs in Figures $2 \mathrm{a}$ and 2c, respectively). Possible integration times were determined by detector software capabilities - five of eight possible settings were selected, based on their ability to collect an image: 1, 2, 4, 8, and 16 seconds. Finally, as shown in Table 1, the SDDs and SODs were organized into nine combinations of locations for X-ray tube and sample stage because these also have essentially infinite combinations.

All possible permutations for selected SDD, SOD, $\mathrm{kVp} / \mathrm{mA}$, and IT were tabulated, resulting in 180 possible combinations. Of these, a random sample of 50 combinations was selected (or a $28 \%$ sample) for parameter testing (see Table 2 for several examples). The metric of Modulation Transfer Function (MTF value, a scale of 0-1) described above (cf. Casali, 2006: Appendix B; Fujita et al., 1992; Pham, 2006), was utilized to calculate image quality. Each test image contained the same potsherd, cut with a diamond coated sectioning saw so that one edge was straight and the MTF could be calculated using an edge-spread function (ESF) (Casali, 2006; Pham, 2006).

To measure MTF, fifty test images were run through a software routine written by the project in Interactive Data Language (IDL), entitled the "Sherd Image Viewer and Analysis" (ShIVA), shown in Figure 3. The program imported imagery, sampled pixel values across the sherd edge, calculated MTF value via fast Fourier Transforms (cf. Fujita et al., 1992; Pham, 2006), and output an MTF measurement for each image and a graph of its distribution. When plotted together, MTF data from these fifty test images suggested optimal

\footnotetext{
${ }^{5}$ Along with the appropriate settings for instrument positions, $\mathrm{kVp}, \mathrm{mA}$, and IT, radiographs must be collected with the aid of "hot" and "cold" frames (also known as "gain' and "offset" images) and "pixel masks," all of which are produced through proprietary detector software. Hot and cold frames, used to normalize each image to the daily geometry and energy variability of the system, are acquired with the same settings as the ultimate radiograph, but without any sample in the region of interest. The hot frame therefore consists of a "blank" image, while the cold frame is acquired without any beam energy. One set of hot and cold images may be used for an entire batch of radiographs and occasionally multiple batches, as long as no spatial or energy settings need to be changed. The pixel mask, which mitigates malfunctioning or failed detector pixels, needs to be acquired less frequently because it will change only as the detector surface degrades over time. It is produced by a proprietary software routine that "maps" the pixel anomalies in the detector, which are eliminated from subsequent imagery.

${ }^{6} \mathrm{It}$ is also important to note that across all systems of radiographic image production, daily and weekly inconsistency in X-ray tube beam output introduces image variability when datasets are acquired over extended time periods (Bushberg et al., 2002; Casali, 2006). This means that regular attention to and measurement of image quality is standard practice across the many fields employing radiography as an evaluative tool.
} 


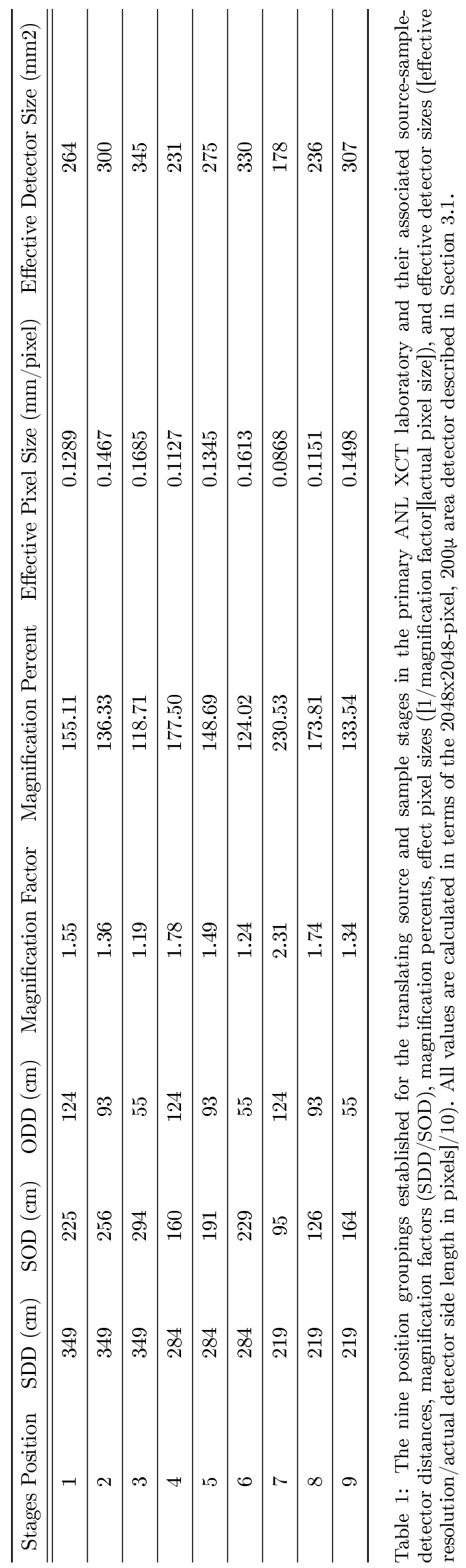




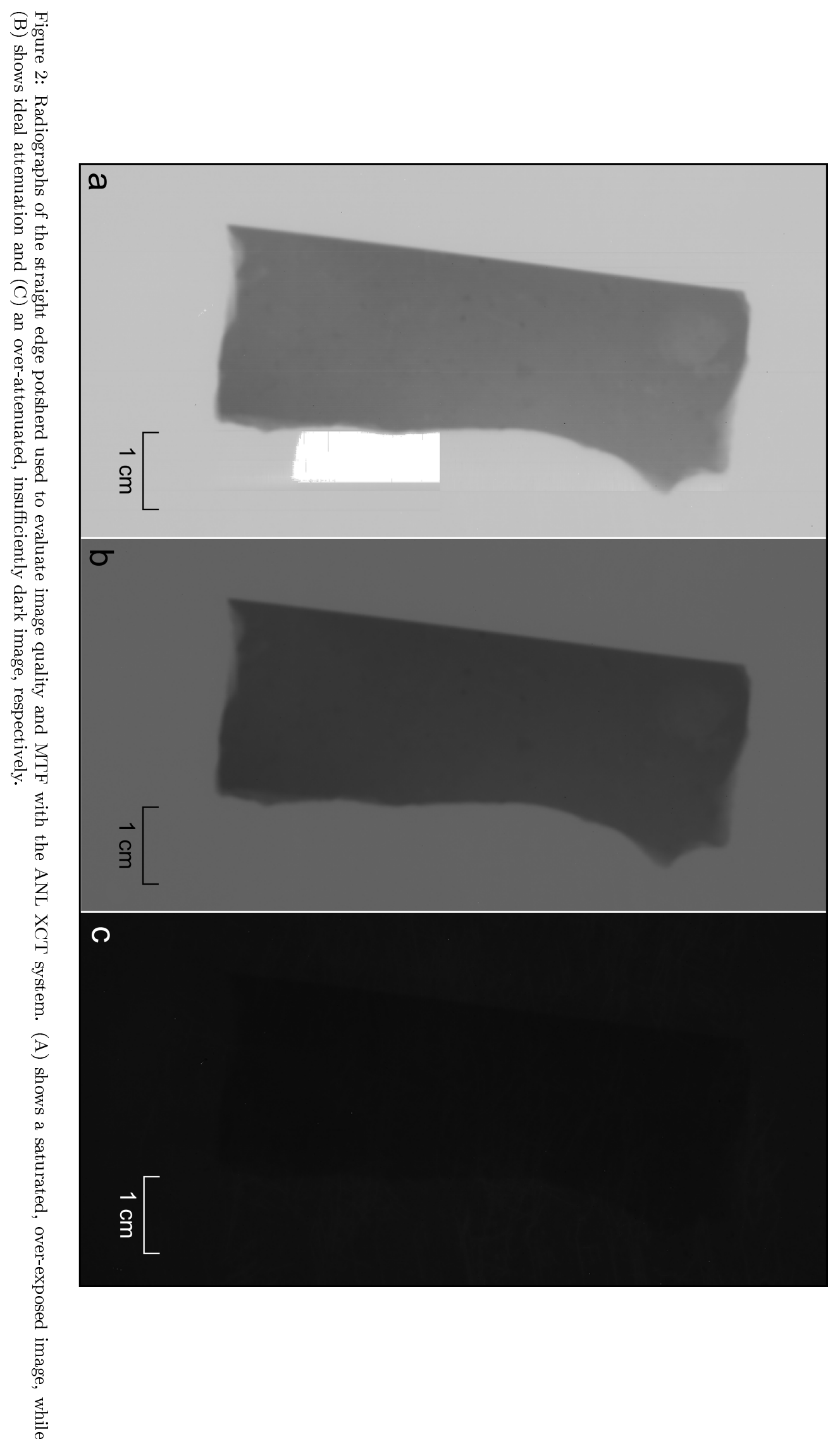




\begin{tabular}{|c|c|c|c|c|c|}
\hline Test \# & Position & $\mathrm{IT}(\mathrm{s})$ & $\mathrm{kVp}$ & MA & MTF \\
\hline 80 & 5 & 1 & 100 & 5.95 & 0.44 \\
\hline 81 & 5 & 2 & 100 & 3.00 & 0.45 \\
\hline 83 & 5 & 8 & 100 & 0.70 & 0.45 \\
\hline 85 & 5 & 1 & 200 & 1.70 & 0.44 \\
\hline 86 & 5 & 2 & 200 & 0.85 & 0.43 \\
\hline 89 & 5 & 16 & 200 & 0.10 & 0.53 \\
\hline 95 & 5 & 1 & 400 & 0.70 & 0.44 \\
\hline 98 & 5 & 8 & 400 & 0.15 & 0.44 \\
\hline 101 & 6 & 2 & 100 & 2.80 & 0.50 \\
\hline 102 & 6 & 4 & 100 & 1.40 & 0.43 \\
\hline 105 & 6 & 1 & 200 & 1.65 & 0.44 \\
\hline 110 & 6 & 1 & 300 & 0.85 & 0.43 \\
\hline 115 & 6 & 1 & 400 & 0.70 & 0.43 \\
\hline 119 & 6 & 16 & 400 & 0.10 & 0.42 \\
\hline 124 & 7 & 16 & 100 & 0.20 & 0.22 \\
\hline 125 & 7 & 1 & 200 & 1.00 & 0.56 \\
\hline 128 & 7 & 8 & 200 & 0.15 & 0.55 \\
\hline 131 & 7 & 2 & 300 & 0.30 & 0.58 \\
\hline 132 & 7 & 4 & 300 & 0.20 & 0.58 \\
\hline 134 & 7 & 16 & 300 & 0.05 & 0.60 \\
\hline
\end{tabular}

Table 2: Groupings of settings parameters for three chosen geometric positions (5-7) and their resultant MTF values when used to image a straight-edge archaeological potsherd. This group is a subset of the 50 unique settings combinations examined during the study. MTF values are presented here as the width of the MTF curve, a Fourier transformation of the Edge Spread Function, at half of its maximum value. 


\begin{tabular}{ccc}
\hline Parameter & Setting & Units \\
\hline \hline Peak kiloVoltage & 275 & $\mathrm{kVp}$ \\
\hline milliAmperes & 0.1 & $\mathrm{~mA}$ \\
\hline Integration Time & 8 & seconds \\
\hline Source-to-Object Distance & close to source & $\mathrm{cm}$ \\
\hline
\end{tabular}

Table 3: Optimal parameters for the DR of archaeological pottery with the ANL system, as determined by examination of the MTF and magnification data.

energy ranges and stage positions, which prompted parameter experimentation with 35 additional settings, focusing most intensively on the 200-300 kV range in setup position seven (see Table 2 for an example of MTF data output. The full dataset is available as a supplementary dataset to the electronic version of this article). The experiments indicated that the following parameters would be ideal for DR of archaeological potsherds in the ANL system: an integration time of 8 seconds, a $\mathrm{kVp}$ between 250 and 275 , an $\mathrm{mA}$ setting of approximately 0.1 to 0.15 , and the positioning of source and object as close together as possible (Table 3 ). These parameters and combinations yield a maximal MTF value above 0.6 and therefore currently offer the highest quality digital radiographs of archaeological potsherds. These parameters also hold true for the larger-pixel, $400 \mu \mathrm{m}$ area detector. Importantly, extensive variability existed across the range of setting permutations, so that highest MTF value was not necessarily the best indicator of preferred settings. For example, while the highest MTF values (>0.6) were achieved at setup positions 3 and 9 (see Table 1), the low level of magnification available in those positions ( $118 \%$ and $177 \%$, respectively) reduced the spatial resolution of sample features overall.

With the aforementioned settings properly configured, assemblage-based (i.e., $\mathrm{n} \geq 300$ ) volumes of potsherd or vessel radiographs may be obtained during a given analytical session. Each image is acquired in less than two minutes. The rate of data collection depends most directly on the amount of time taken to switch samples and complete safety checks. MAE has collected well over 100 radiographs in a single day's session. Following initial acquisition, radiographs are post-processed via computational operations in ShIVA that allow extraction of useful parameters for statistical analysis and pattern definition.

\subsubsection{Image Processing Protocols}

Following data acquisition, radiographs are submitted through a sequence of transformations, manipulations, and quantifications that: equalize image histograms, stretching collected pixel values over the full dynamic range of the X-ray detector; create third-order, "filtered" depictions relying on IDL algorithms to analyze data variability; and extract specific data parameters for analysis (e.g., frequency, density, shape, etc. of voids, inclusions, and matrix gradients). These operations are performed through dedicated data entry tabs in ShIVA (see Figure 3).

First, batch transformations prepare imagery for secondary image production. The 16-bit proprietary raw format used by the detector software ("HIS" format) is initially converted to the common "TIFF" image format. Users are then able to optionally process each radiograph to use the complete 8-bit (0-255) dynamic range of their display through histogram equalization (see Casali, 2006; Lang and Middleton, 2005). Since each potsherd may take up less than half of the full image area (i.e., detector), even under maximum magnification, the range of pixel values within the sherd is often less than the full dynamic range of the resultant radiograph. This difference between "background" and sample can be leveraged by using histogram equalization to improve the overall contrast and permit better visualization of the sample's internal structures.

The extent of the sample is first determined using a region-growing technique (Russ, 2011: 439). The analyst selects a single point within the sherd or vessel and the software identifies adjacent pixels within a range of gray-levels as also being part of that sample. This process is iteratively repeated with an increasing range of gray-levels until the region bleeds into the background (identified by a very large increase in area of the region in a single iteration). Processed images are then created by expanding the grayscale range within the sherd to the full dynamic range of the image (using 10- and 8-bit formats). These processed images and the $\mathrm{x}$-y region identified as being part of the potsherd are saved for subsequent analysis. Note that the original image pixel values are still available and used in subsequent image analysis and coding (see Section 


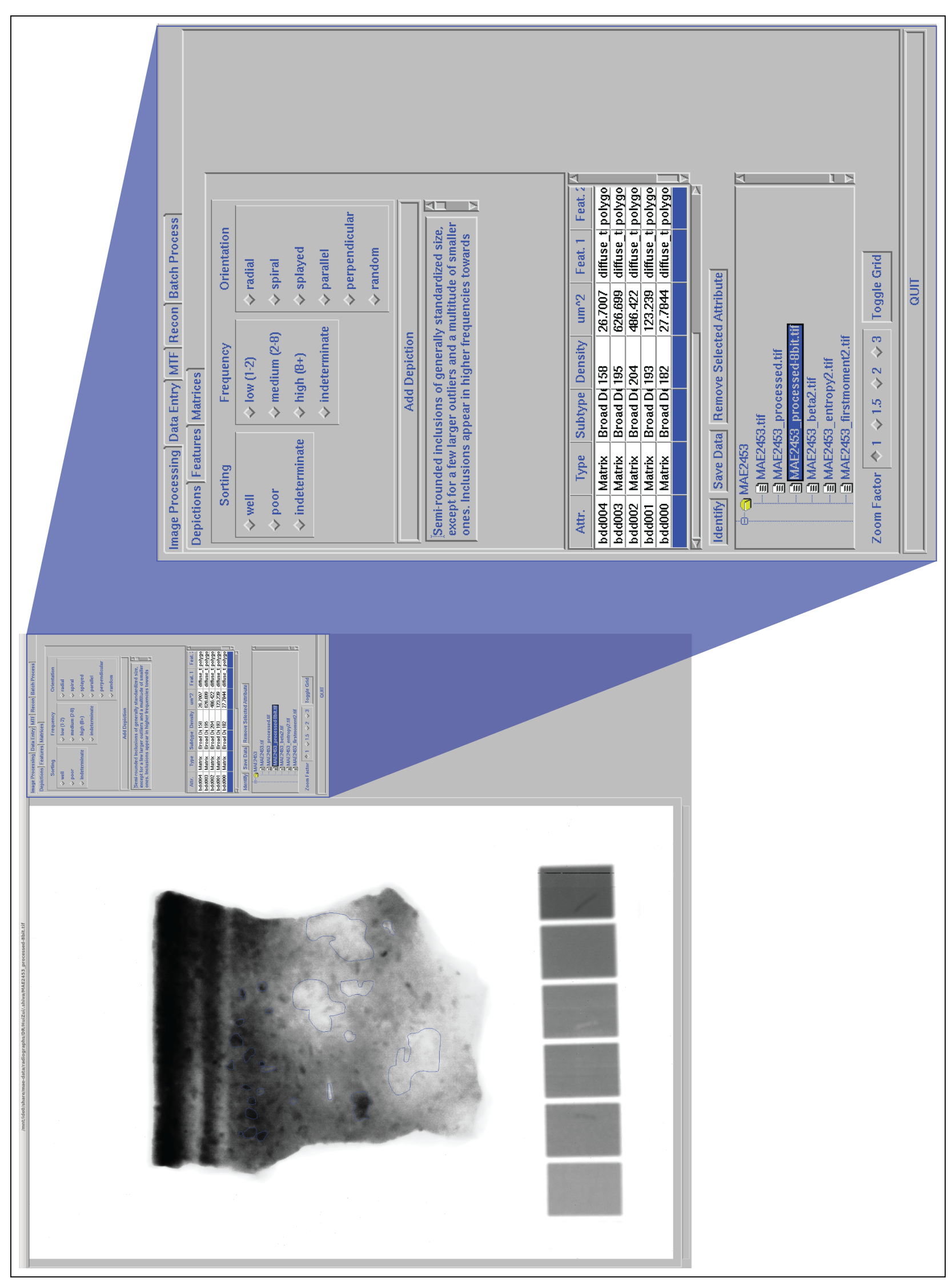

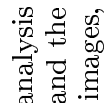

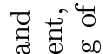

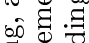

空

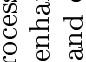

iิ

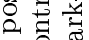

诺

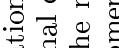

需.

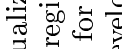

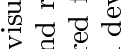

菏.:

요의

跣语

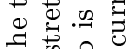

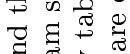

สี

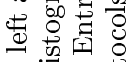
․․ㄹ

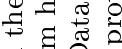

ริ

客苛专

둥 क्ष

के है

교

.

.$\Xi \widetilde{\pi}$

\&.

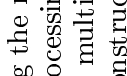

.

s.

क है

这电

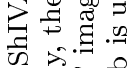

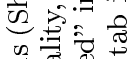

吕

疍

च

荡通.

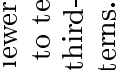

$>$ D

so

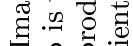

휴유

巧

记是要

\%

苋䨔

壳

行

F

$\ddot{\circ} \exists \overline{0}$

$\stackrel{9}{\leftrightarrows} \frac{7}{0} \cdot \frac{9}{3}$ 社范范 
4 below).

For example, in the imagery from MAE sample 2453, a guan jar sherd from the Longshan period site of Huizui, China (Figure 4a), equalization reveals stark contrasts in the comparative density of inclusions, specific pore areas, and lower density sectors (similar to traditional Xeroradiography) that represent the impact zones characteristic of the paddle and anvil formation technique used in guan jar construction (Figure 4b). While paddle and anvil forming was previously understood to play a role in Longshan pottery production (Li and Huang, 1993), the digital radiography as processed, viewed, and analyzed in ShIVA enables a statistical evaluation of the technique (impact zone size, density of zones, location of extant zones in vessel well, etc.) and makes possible, for example, a regional examination of this formation technique's application in the Late Neolithic, examining the spatial differentiation of various production systems across Longshan sites and landscapes. Assemblage based DR has enabled Hartley's research to distinguish a diachronic contrast between conservation of paste preparation and formation techniques on the one hand and dynamism in surface treatment and decoration regimens on the other. (See the article by Greene and colleagues [n.d.], currently in preparation, for the full case study of the Longshan materials from Huizui.)

Equalization also reveals an agglomeration of higher density inclusions in the rim and neck portions of the sample. This configuration may represent: (1) a secondary effect of the paddle and anvil technique that pushed large aplastic inclusions toward the top of the vessel as it thinned and extended the wall vertically or (2) the presence of an applied rim, neck, or both, assembled from a paste containing aplastic inclusions with both a significantly tighter spatial distribution and higher average density. The lack of visible gradient transitions in the upper portion of the vessel, however, suggest that applied elements are less likely responsible. The digital aspect of the data allows continuing research to measure the distribution frequencies and radiodensities of these features as an evaluation of paddle and anvil technique complementary to the impact zone study noted above.

Normalized data files like that in Figure $4 \mathrm{~b}$ are output in both 8- and 16-bit format. 16-bit images are subsequently manipulated in ShIVA to produce color imagery using five different filtering algorithms available in IDL (Figures 4c and 4d). Drawing on data richness beyond the capacities of human visual perception, these filters compute features related to local texture and gradient of small regions of interest (ROIs) over the entire sample area. Texture refers to the spatial distribution of gray-level values in the image or ROI, while gradient refers to directional trend in the same values (Russ, 2011: 297-305). Analyzed texture features include the Fourier-based first moment of the power spectrum (a measure of texture coarseness or fineness), the root-mean-square (RMS) variation (a measure of texture magnitude), and the amount of randomness of the gray-levels in the image ("entropy" calculation), as well as fractal-based texture features (Russ, 2011: 312-317). Texture values are converted to color images using two scales: (1) the entire color table spectrum for data present in an individual image and (2) a fixed scaling that correlates identical texture values and features across all images.

Each filter emphasizes different aspects of inclusions and matrices ("texture" and "gradient") (Casali, 2006; Quinn and Sigl, 1980), so their analytical usefulness varies according to the sample. In the Longshan example (Figure 4, MAE sample 2453) the "Beta fractal" filter shows in fine detail the density distributions of inclusions, revealings blue-coded particles featuring higher densities gathered toward the upper portion of the guan jar during vessel formation (Figure 4c), a third-order analytical result that manipulates digital pixels to effect a statistically-based, visual illustration of patterning that is not visible in the raw or equalized radiographs. This kind of fabric evaluation is similar to the manner in which X-radiographic instruments were manipulated to emphasize particle density differences (Carr, 1990, 1993; Carr and Riddick, 1990; Rye, 1977). On the other hand, entropy filtering helps to sharpen and delineate more precise boundaries for the three, dark orange paddle impact zones in the sample (Figure 4d), another third-order result. Thus, ShIVA's various post-processing and filtering modules can both draw out fundamental features and matrix patterns and also leverage the digital image format to achieve analyses that were never approached with analog instrumentation. On an assemblage scale, these tools enable a quantitative impact that can be used to distinguish between different techniques of paste preparation and formation, but also, at this high resolution, smaller scale factors of difference that can point to distinctions between individual potters, individual "batches" of pottery production, or vessels crafted with distinct individual potting tools.

Following the production of secondary imagery, such as the beta and entropy conversions in Figures $4 \mathrm{c}$ and d, data entry modules in ShIVA are used to tag or "code" images through visual analysis of regional features and pixel variability. Analysts encircle and describe individual features of interest using a mouse, trackball, 


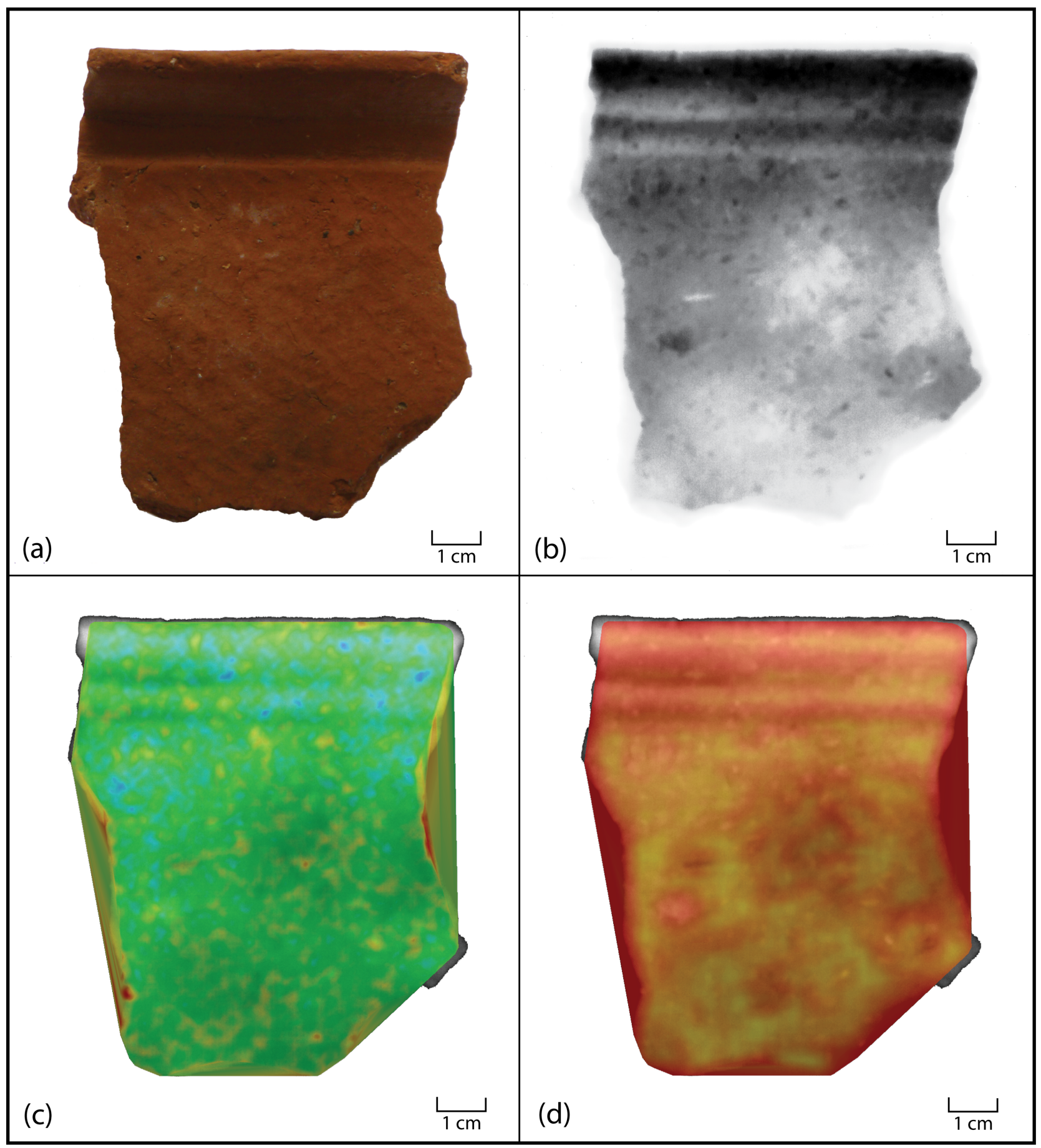

Figure 4: MAE sample 2453, a rim and shoulder potsherd from a guan jar recovered during the excavation of the Longshan site of Huizui, China (third millennium BC). (A) displays a photograph of the sample and (B) the histogram-stretched, "equalized" radiograph. Subsequent manipulation through regional manipulation algorithms revealed that the "beta fractal" image seen in (C) isolates the concentrations of inclusions pushed upwards in the vessel wall through paddling action; and the "entropy" filter image seen in (C) is productive for distinguishing paddle-and-anvil formation and delineating paddle impact zones. 
stylus, or other input device. Collected into an integrated relational database (or output as text files), resultant data consist of parameter values drawn from visual pottery analysis, geoarchaeological sediment study, and matrix phenomena first identified by Rye (1977) then subsequently applied by numerous others (Berg, 2008, 2009; Carr, 1990; Heinsch and Vandiver, 2006; Vandiver, 1987, 1988) in radiographic analyses of archaeological pottery.

\section{Analyzing Large Potsherd DR Datasets with Digital Tools}

The specific data collection, category attribution, and pattern definition activities facilitated by ShIVA connect our IDL image analysis software to a PostgreSQL- and Python-based database run on the same UNIX platform. Hence, users can define multi-scale image regions they deem significant to a given sample, record them into the database, and assign qualitative and quantitative values. These data become available in tabular format for statistical description and analysis, and facilitate an efficient process of pattern definition and classification across large volumes of complex pottery imagery.

\subsection{Extracting Qualitative and Quantitative Datasets}

Each image opened in the viewer is normalized for spatial resolution (effective pixel size) within the program, while they are scaled for varying sample thickness outside of ShIVA, through $\mathrm{R}$ statistical software (cf. Carr, 1993; Carr and Riddick, 1990). The ShIVA data entry module (see Figure 3) provides several basic viewing tools to facilitate analysis. Scaling controls allow zooming between magnification levels and a five-millimeter grid can be toggled over the viewer to help define spatial sub-sections. The "Depictions" sub-tab allows the analyst to record variables describing sorting quality, inclusion frequency, and dominant inclusion orientation of the overall image, as well as narrative notes.

Additional data collection can be initiated from the right uppermost portion of the interface (see Figure 3 ) in the form of defining regions for four types of ceramic sub-structures: "inclusions," "voids," "broad density differences," and "distinct boundaries."7 While the former-voids (fabric pores) and inclusions (aplastic constituents)--are familiar parameters among pottery analysts, "broad density differences" and "distinct boundaries" were developed to describe the types of features visible as coherent matrix gradients in radiographic data sets. While "distinct boundaries" refer to clearly defined and sharply delimited sub-structures, "broad density differences" denote amorphous or subtle gradient transitions (see Table 4). All four terms refer to pottery structures of different scales and characters, parameterized by us in terms of their representation in radiographic imagery.

An analyst uses a mouse or trackball to sequentially outline features for dataset import. Each is given an alphanumeric string and listed in the data table. Individual inclusions or voids can be highlighted by clicking on their row in the right-hand table. Along with simple vector outlines, each feature is recorded for standard sedimentary variables of "roundness," "sphericity," "angularity," or "shape," and "notes," consisting of narrative description. "Size" (total feature area in square microns), "grain type" (derived from the "size" variable), and "[radio]density" (calculated from the average pixel values of the feature) are determined programmatically by the application, normalizing for spatial resolution as noted above, and added to the feature's associated data. The coded inclusions and voids for the Longshan samples (MAE 2453) can be seen in Figure 3 as variously shaped and sized features that have been digitally outlined and measured for roundness, sphericity, surface area, etc. The resultant qualitative and quantitative data collected for each feature and imported to the dataset can be reviewed in the table located on the right side of the viewer. A full account of current variable options and values is listed in Table 4.

The matrix gradient structures "broad density difference" and "distinct boundary" range from straightforward interpretation as construction coils, slab joins, fingerprints, or paddle impact zones, to more challenging identification such as different paste combinations, applied vessel portions, chaff burnouts, firing cracks, or well-blended joins. Figure 3 shows the aforementioned paddle impact zones for MAE sample 2453 outlined as broad density differences, the resultant surface areas and average densities for each added to the dataset

\footnotetext{
${ }^{7}$ ShIVA software can be downloaded at http://mae.stanford.edu/ShIVA.php; run on PC, Apple, or Linux platforms; and configured to output data to SQL-based databases or text files.

${ }^{8}$ Since these same variables are useful for distinguishing low-density inclusions from actual voids, they are recorded for both types of feature.
} 


\begin{tabular}{cc}
\hline Parameters by Sub-tab & Values \\
\hline Depictions Tab (Overall Image) & well, poor, indeterminate \\
\hline Sorting & low, medium, high, indeterminate \\
\hline Frequency (per 5mm grid square) & random \\
\hline Orientation & \\
\hline Features Tab (Voids and Inclusions) & $0.1,0.3,0.5,0.7,0.9$ \\
\hline Roundness & $0.1,0.3,0.5,0.7,0.9$ \\
\hline Sphericity & $\begin{array}{c}\text { polygonal, splayed, parallel, perpendicular, } \\
\text { Shape }\end{array}$ \\
\hline Angularity & rectangular, oval, circular square, triangular, \\
\hline Area & angular, sub-angular, sub-rounded, rounded \\
\hline Density & programmatic calculation (mm2) \\
\hline Grain size & programmatic calculation (16-bit pixel values) \\
\hline Talt, very fine sand, fine sand, medium sand, \\
coarse sand, very coarse sand, granules, pebbles \\
\hline Matrices (Broad Density \\
and Distinct Boundaries)
\end{tabular}

Table 4: The array of ShIVA data entry parameters collected by the software at present and the potential values for each. Data entry is conducted through three tabs: Depictions (characterizations of the entire sherd/image), Features (characterizations of inclusions and pores/voids), and Matrices (characterizations of gradients visible in the clay matrix). Parameters in italics are calculated by the software, not entered by the user. 
visible on the right of the interface. As image coding continues and more analysts become familiar with digital radiographic data from archaeological pottery (see Greene et al., n.d.), these subtypes will undergo refinement.

\subsection{Descriptive and Image-derived Data Side-by-Side}

All of the aforementioned data formats are collected in the articulated PostgreSQL database, which is connected to $\mathrm{R}$ statistical software through the language $\mathrm{PL} / \mathrm{R}$, allowing all manner of preferred statistical descriptions and manipulations (Conway, 2008; Singh et al., 2008). In this way, ShIVA is used to extract and analyze qualitative and quantitative data at several scales: (1) individual images as representations of entire potsherds; (2) inclusion and void structures radiographically visible in the sample fabric; and (3) matrix features visible radiographically as gradients at either the "equalized" (Figure $4 \mathrm{~b}$ ) or filtered image (Figures $4 \mathrm{c}$ and $4 \mathrm{~d}$ ) stage. Features and sub-structures, such as consistent inclusion, void, and matrix patterns, can be examined and summarized within individual samples and compared across a large-scale dataset. Indeed, this form of extensive post-facto statistical examination is indispensable for the second and third order data made possible by digital radiographic image analysis, as an analyst working with ShIVA moves quickly between multiple structural scales and samples, and retains only an impressionistic sense of the complete dataset. Case studies of pottery assemblages from three of MAE's study areas in Eurasia, described elsewhere (Doumani, 2014; Greene, 2013; Greene et al., n.d.), have drawn on both quantitative and qualitative aspects of the resultant data to present arguments about their various pottery traditions and the societies within which they were situated. These studies, in preparation as a separate article by Greene and colleagues (n.d.), focus on topics ranging from Greene's work on the economic life of second millennium BC polities in the South Caucasus, to Hartley's aforementioned study of communities of potting practice and social difference in Late Neolithic China, to Doumani's examination of local potting and foodways and their relationship to traditional culture histories of Bronze Age Central Asia. The research demonstrates the diverse range of contributions that DR data sets are poised to make in the analysis of pottery production and the broader study of the social import of ceramic materials.

\section{Conclusion: A Programmatic Digital Radiography for Archaeological Science}

Structural investigations of pottery manufacture through DR-examining topics as diverse as detailed sequences of production techniques, overwrought technological models, and frozen culture histories - can push beyond the first-order, qualitative analysis employed in analog radiography and establish archaeological ceramic radiography as a true quantitative counterpart to contemporary geochemical and mineralogical approaches. Such studies are also able to fill gaps left by compositional characterization and by destructive approaches that rely on smaller, less representative assemblages. DR systems and software tools like ShiVA permit the simultaneous qualitative and quantitative study of structures (1) at both macro- and micro-scales and (2) across greater portions of potsherds and vessels than is possible with thin-sections, powdered samples, or surface analyses. While ShIVA currently requires a human analyst to directly supervise image postprocessing and coding for each individual radiograph, future enhancements will target computer-facilitated and more autonomous image processing, speeding up the analytical process even further and allowing for an expanded number of evaluative criteria.

Beyond the analytical benefits of assemblage-based capacity, quantification, and whole sample analysis, DR retains those basic, positive elements that have characterized radiographic approaches since early applications of X-rays in art and archaeology: it is a non-destructive method without short or long term impacts to samples or analysts. Non-destructive benefits extend to the geometric and logistical aspects of the imaging apparatus as well. Because experimental, industrial radiographic equipment is not contained in a cabinet or limited by a sample holder, samples of any size - from enormous storage pithoi to small "model" vessels and potsherds - can be imaged, permitting analysis of more representative assemblages (cf. Shennan, 1997: 364).

To take full advantage of these benefits, utilization of consistent protocols and image quality monitoring are paramount. MAE continues its data collection within the protocol ranges listed in Section 3.2 above, but it is important to note that these settings are ranges of the broader spectrum available to the analyst. Exact optimum parameters need to be determined for the specific radiographic system employed, depending heavily on source energy capacity, detector speed and resolution, and source, object, and detector geometric configuration. Thus, dependable software for testing image quality and consistency is of equal importance 
to high-contrast and high-resolution imagery. MAE's research has thus concentrated equally across protocol development in data acquisition, post-processing, and image analysis, with ShIVA now available for free distribution. ${ }^{9}$

While DR tools for materials analysis represent powerful and essential steps forward in the structural materials analysis of pottery, prospects for XCT study in three dimensions are an essential next stage in radiographic technique development. As described, DR imagery compresses the full sample thickness into a two-dimensional view, leaving little sense of feature depths. Moreover, overlapping sub-structures can be conflated during feature analysis and obscure one another in the eye of the analyst. By providing a threedimensional view instead, XCT "tomogram" data avoids these drawbacks and provides the analyst with an unprecedented ability to peer through the sample from an essentially unlimited range of geometries. Digital "longitudinal" sectioning of 3D CT data sets, drawing directly on the research of Rye and his Xeroradiographer and X-radiographer contemporaries (Carr, 1990, 1993; Carr and Riddick, 1990; Vandiver, 1987, 1988), would provide particularly powerful views of paste preparation and formation, increasing the level of feature identification far beyond the capabilities of DR. MAE's technique development towards systematic XCT protocols is currently in progress.

The increased level of DR application and technique development described above cannot be performed within current deployments of radiographic infrastructure alone. While DR advancements constitute an important move forward from obsolete and increasingly inaccessible analog instruments, well-maintained experimental DR/XCT laboratories featuring more "open" industrial systems remain rather small in number. New archaeometric infrastructures will need to be assembled in order to support both the use of existing methods and the development of directed improvements and appropriate customizations for archaeological materials. In particular, this will reduce the current dependence on medical apparatuses, which in our experience between ANL and the Washington University School of Medicine in St. Louis (Doumani pers. comm.) are both comparatively expensive to use and not well configured to accommodate the variable geometries of archaeological pottery. The MAE project's collaborative efforts at ANL can serve as a model for ongoing research at state-run scientific campuses, but they can also be used to model the development of academic laboratories dedicated to digital archaeological radiography. The organization of permanent DR and XCT analytical facilities will be essential to the promulgation of the method for pottery analysis and its extension to analyze other classes of material culture.

\section{Acknowledgements}

The authors owe their gratitude to two primary groups of colleagues, without which the technique development described in this article would not have been possible. Founding MAE Project members MaryFran Heinsch, Lori Khatchadourian, David Peterson, and Adam T. Smith were central to the development of the research plan that allowed the crafting and testing of DR imaging protocols. Collaborators Christopher Deemer, William Ellingson, and Richard Koehl at Argonne National Laboratory provided critical technical expertise, guidance, and on-site support that assured our daily efforts at the XCT laboratory would be collaborative and efficient. In addition, programmer Alexander Vostrikov played a key role in the creation of our relational, SQL database and its integration into the ShIVA application. The support of the Argonne Board of Governors and the University of Chicago Research Office was also critical to providing our project with an initial footing and impetus toward research. Finally, thanks are owed to the many colleagues and institutions that supported this research or aided in the recovery and provision of archaeological materials for DR analysis. They include Pavel Avetisyan, Ruben Badalyan, Wang Facheng, Michael Frachetti, Li Liu, Alexei Mar'yashev, Anne Underhill, Chen Xingcan, Li Yongqiang, and the Armenian Institute of Archaeology and Ethnography, the Dolores Zohrab Liebmann Fund, the Institute of Archaeology Kazakhstan in Almaty, Lambda Alpha, the Luce Foundation/American Council of Learned Societies, the National Science Foundation, the University of Chicago Department of Anthropology, the U.S. Department of Education, the Washington University St. Louis Department of Anthropology, and the Wenner-Gren Foundation. We are also grateful to Adam T. Smith and Marie-Claude Boileau for reading initial drafts of this paper. This material is based upon work supported by the National Science Foundation under Grant No. 0808151.

\footnotetext{
${ }^{9}$ http://mae.stanford.edu/ShIVA.php
} 


\section{References}

Applbaum, N., Applbaum, Y. H., 2005. The Use of Medical Computed Tomography (CT) Imaging in the Study of Ceramic and Clay Artifacts from the Ancient Near East. In: Uda, M., Demortier, G., Nakai, I. (Eds.), X-rays for Archaeology. Springer, Dordecht, The Netherlands, pp. 231-245.

Berg, I., 2008. Looking through pots: recent advances in ceramics X-radiography. Journal of Archaeological Science 35 (5), 1177-1188.

Berg, I., 2009. X-radiography of Knossian Bronze Age vessels: assessing our knowledge of primary forming techniques. The Annual Review of the British School at Athens 104.

Berg, I., 2011. Exploring the chaîne opératoire of ceramics through X-radiography. In: Scarcella, S. (Ed.), Archaeological ceramics: a review of current research. BAR International Series 2193. Archaeopress, Oxford, pp. 57-63.

Boyce, S. J., Samei, E., 2006. Imaging properties of digital magnification radiography. Medical Physics 33 (4), 984-996.

Braun, D. P., Jul. 1982. Radiographic Analysis of Temper in Ceramic Vessels: Goals and Initial Methods. Journal of Field Archaeology 9 (2), 183-192.

URL http://www.jstor.org/stable/529476

Brennan, J., 2002. An introduction to digital radiography in dentistry. Journal or Orthodontics 29 (1), 66-69.

Bushberg, J. T., Seibert, J. A., Leidholdt, Jr., E. M., Boone, J. M., 2002. The essential physics of medical imaging, 2nd Edition. Lippincott Williams \& Wilkins, Philadelphia.

Carr, C., 1990. Advances in ceramic radiography and analysis: applications and potentials. Journal of Archaeological Science 17 (1), 13-34.

Carr, C., Jan. 1993. Identifying Individual Vessels with X-Radiography. American Antiquity 58 (1), 96-117. URL http://www.jstor.org/stable/281456

Carr, C., Komorowski, J. C., 1995. Identifying the mineralogy of rock temper in ceramics with X-radiography. American Antiquity 60 (4), 723-749.

Carr, C., Riddick, E. B., 1990. Advances in ceramic radiography and analysis: laboratory methods. Journal of Archaeological Science 17 (1), 35-66.

Casali, F., 2006. X-ray and neutron digital radiography and computed tomography for cultural heritage. In: Bradley, D., Creagh, D. (Eds.), Physical techniques in the study of art, archaeology, and cultural heritage. Vol. 1. Elsevier, Amsterdam, pp. 41-123.

Conway, J. E., 2008. PL/R - R probabilismcedural langugage for Postrepro.

URL http://www.joeconway.com/plr/

Curry III, T. S., Dowdey, J. E., Murry, Jr., R. C., 1990. Xeroradiography. In: Curry, T. S., Dowdey, J. E., Murry, R. C. (Eds.), Christensen's physics of diagnostic radiology, 4th Edition. Lea and Febiger, Philadelphia, pp. 266-288.

Davis, R., 2005. Radiography: Archaeo-Human and Animal Remains. Part I: Clinical Radiography and Archaeo-Human Remains. In: Lang, J., Middleton, A. (Eds.), Radiography of cultural material. Elsevier Butterworth-Heinemann, Burlington, MA, pp. 130-149.

Doumani, P. N., 2014. Bronze Age potters in regional context: long-term development of ceramic technology in the Eastern European steppe zone. Ph.D. thesis, Washington University in Saint Louis.

Ellingson, W. A., Koehl, E. R., Engel, H. P., Sun, J. G., Deemer, C., 1997. Development of non-destructive evulation methods for structural ceramics. In: Judkins, R. R. (Ed.), Proceedings of the eleventh annual conference on fossil energy materials. Oak Ridge National Laboratory, Oak Ridge, TN, pp. 79-87. 
Ellingson, W. A., Vandiver, P. B., Robinson, T. K., Lobick, J. J., 1988. Radiographic Imaging Technologies for Archaeological Ceramics. Tech. rep., Argonne National Laboratory, Argonne, Illinois.

Foster, G. V., 1985. Identification of inclusions in ceramic artefacts by ceramic X-radiography. Journal of Field Archaeology 12 (3), 373-376.

Fujita, H., Tsai, D.-Y., Itoh, T., Doi, K., Morishita, J., Ueda, K., Ohtsuka, A., 1992. A simple method for determing the modulation transfer function in digital radiography. IEEE Transactions on Medical Imaging 11 (1), 34-39.

Glanzman, W. D., Fleming, S. J., 1986. Xeroradiography: a key to the nature of technological change in ancient ceramic production. Nuclear Instruments \& Methods in Physics Research, Section A: Accelerators, Spectrometers, Detectors, and Associated Equipment 242 (3), 588-595.

Gonzalez, R. C., Woods, R. E., 2007. Digital image processing, 3rd Edition. Prentice Hall, Upper Saddle River, NJ.

Greene, A. F., 2013. The social lives of pottery on the Plain of Flowers: an archaeology of pottery production, distribution, and consumption in the Late Bronze Age South Caucasus. Ph.D. thesis, The University of Chicago.

Greene, A. F., In Press. Synchrotron radiation. In: Hunt, A. M. W. (Ed.), The Oxford handbook of archaeological ceramic analysis. Oxford University Press, Oxford.

Greene, A. F., Hartley, C. W., 2009. From Analog To Digital: protocols And Program For A Systematic Digital Radiography Of Archaeological Pottery. In: Biró, K. T., Szilágyi, V., Kreiter, A. (Eds.), Vessels inside and outside: proceedings of the conference EMAC '07 9th European Meeting on Ancient Ceramics, 24-27 October 2007. Hungarian National Museum, Budapest, pp. 5-14.

Greene, A. F., Hartley, C. W. (Eds.), n.d. Renewing the search for structure: applying myltiple scales and instruments in the analysis of production. Equinox Publishers, London.

Greene, A. F., Hartley, C. W., Doumani Dupuy, P. N., n.d. X-ray perspectives: structural analyses of ancient Eurasian pottery accomplished with digital radiography.

Heinsch, M., Vandiver, P., 2006. Recent Xeroradiographic Analysis of Kura-Araxes Ceramics. In: Peterson, D. L., Popova, L., Smith, A. T. (Eds.), Beyond the steppe and the sown: proceedings of the 2002 University of Chicago Conference on Eurasian Archaeology. Brill, Leiden, pp. 382-394.

Hellier, C. J., 2012. Handbook of nondestructure evaluation, 2nd Edition. McGraw-Hill, New York.

Herring, W., 2011. Learning radiology: recognizing the basics, 2nd Edition. Elsevier Mosby, Philadelphia.

Ishida, M., Dol, K., Loo, L. N., Metz, C. E., Lehr, J. L., 1984. Digital image processing: effect of detectability of simulated low-contrast radiographic patterns. Radiology 150 (2), 569-577.

Jakes, K. A. (Ed.), 2002. Archaeological chemistry: materials, methods, and meaning. Oxford University Press.

Kahl, W.-A., Ramminger, B., 2206-2219 2012. Non-destructive fabric analysis of prehistoric pottery using high-resolution X-ray microtomography: a pilot study on the late Mesolithic to Neolithic site HamburgBoberg. Journal of Archaeological Science 39 (7).

Kreit, E., Mathger, L. M., Hanlon, R. T., Dennis, P. B., Naik, R. R., Forsythe, E., Heikenfeld, J., 2013. Biological versus electronic adaptive coloration: how can one inform the other? Journal of the Royal Society Interface $10(78)$.

Lambert, J., 1997. Traces of the past: unraveling the secrets of archaeology through chemistry. Perseus Books, Massachusetts. 
Lang, J., 2005. Metals. In: Lang, J., Middleton, A. (Eds.), Radiography of material culture, 2nd Edition. Elsevier Butterworth-Heinemann, Oxford, pp. 51-75.

Lang, J., Middleton, A. (Eds.), 2005. Radiography of cultural material, 2nd Edition. Elsevier ButterworthHeinemann, Oxford.

Lechtman, H., Parsons, L. A., Young, W. J., 1975. Seven matched hollow gold jaguars from Peru's early horizon. No. 16 in Studies in Pre-Columbian Art and Archaeology. Dumbarton Oaks, Trustees for Harvard University, Washington, D. C.

Li, W., Huang, S., 1993. Study of the pottery-making technology in the Yellow River Basin [Huang He Liuyu Xinshiqi Shidai Zhitao Gongju de Yanjiu]. Huaxia Kaogu 3, 66-87.

Liaptsis, D., Cooper, I., Ludford, N., Gunner, A., Williams, M., Willis, D., Roberson, C., Falticeanu, L., Brown, P., 2010. NDT characterisation of boron carbide for ballistic applications. In: Mathur, S., Ohji, T. (Eds.), Advances in ceramic armor VI: ceramic engineering and science proceedings. Vol. 31. WileyBlackwell, Hoboken, NJ, pp. 25-36.

Mei, H., Xu, Y., Cheng, L., Zhang, L., 2007. Nondestructive evaluation and mechanical characterisation of a defect-embedded ceramic matrix composite laminate. International Journal of Applied Ceramic Technology 4 (4), 378-386.

Middleton, A., 2005. Ceramics. In: Lang, J., Middleton, A. (Eds.), Radiography of cultural material, 2nd Edition. Elsevier Butterworth-Heinemann, Oxford, pp. 76-95.

Pham, T., 2006. Spatiotonal adaptivity in superresolution of undersampled image sequences. Ph.D. thesis, Delft University of Technology.

Pollard, M., Heron, C., 2008. Archaeological chemistry, 2nd Edition. Royal Society of Chemistry, Cambridge.

Quinn, R. A., Sigl, C. C. (Eds.), 1980. Radiography and modern industry, 4th Edition. Eastman Kodak Company, Rochester, NY.

Röntgen, R., 1896. On a new kind of rays. Nature 53, 264-276.

Russ, J. C., 2011. The image processing handbook, 6th Edition. CRC/Taylor and Francis, Boca Raton, FL.

Rye, O. S., 1977. Pottery Manufacturing Techniques: X-ray Studies. Archaeometry 19 (2), 205-211.

Rye, O. S., 1981. Pottery technology: principles and reconstruction. No. 4 in Manuals on archeology. Taraxacum, Washington, D.C.

Samei, E., Flynn, M. J., Reimann, D. A., 1998. A method for measuring the presampled MTF of digital radiographic systems using an edge test device. Medical Physics 25, 102-113.

Shennan, S., 1997. Quantifying Archaeology. Edinburgh University Press, Edinburgh.

Singh, S., Mayfield, C., Mittal, S., Prabhakar, S., Hambrusch, S., Shah, R., 2008. Orion 2.0: native support for uncertain data. In: Lakshaman, L. V. S., Ng, R. T., Shasha, D. (Eds.), Proceedings of the 2008 ACM SIGMOD international conference on Management of data. Association for Computing Machinery, New York, pp. 1239-1242.

Sun, J. G., Petrak, D. R., Pillai, T. A. K., Deemer, C., Ellingson, W. A., 2008. Nondestructive evaluation and characterisation of damage and repair for continuous fiber ceramic composite panels. In: Bray, D. E. (Ed.), 22nd annual conference on composites, advanced ceramics, materials, and structures: A: ceramic engineering and science proceedings. Vol. 19. American Ceramic Society, Westerville, OH, pp. 615-622.

Vandiver, P., 1987. Sequential Slab Construction; A Conservative Southwest Asiatic Ceramic Tradition, ca. 7000-3000 B.C. Paléorient 13 (2), 9-35. 
${ }_{663}$ Vandiver, P., 1988. The Implications of Variation in Ceramic Technology: the Forming of Neolithic Storage

664 Vessels in China and the Near East. Archaeomaterials 2, 139-174.

665 Vandiver, P., Ellingson, W. A., Robinson, T. K., Lobick, J. J., Seguin, F. H., 1991. New Applications of 666 X-Radiographic Imaging Technologies for Archaeological Ceramics. Archaeomaterials 5 (2), 185-207.

${ }_{667}$ Yoder, D. T., 2008. The Use of "Soft" X-ray Radiography in Determining Hidden Construction Characteristics ${ }_{668} \quad$ in Fiber Sandals. Journal of Archaeological Science 35 (2), 316-321. 